\title{
Demo Abstract: Service Oriented Wireless Sensor Networks - A Cluster-based Approach
}

\author{
Raluca Marin-Perianu, Hans Scholten and Paul Havinga \\ University of Twente \\ Enschede, The Netherlands \\ Email: r.s.marinperianu@utwente.nl
}

\section{INTRODUCTION}

Wireless Sensor Networks (WSNs) have recently emerged as a viable technology for various industrial and business applications [4]. However, WSNs still lack a uniform mechanism of exposing their functionality to the user. In this sense, service-based solutions represent a well-suited abstraction [2], as they provide an easy-touse interaction paradigm and decouple the application from the low-level technologies.

In this paper we demonstrate a service-oriented solution for heterogeneous WSNs. The main operations are service discovery and service usage. In addition, our solution is integrated with mobile platforms (smartphones and PDAs), that act as gateways to the GSM network and the Internet.

For energy-efficiency reasons, the service discovery protocol is based on a clustering structure, which facilitates the construction of a distributed directory of services available in the network [5]. The clusterheads (or root nodes) keep a registry for all the services in their clusters. During the discovery phase, the service discovery messages are disseminated only among the root nodes, thus preventing network flooding. The clustering protocol is designed to: (1) make decisions only based on 1-hop neighbourhood information, (2) avoid the chain reactions (where a single topology change in the network may trigger significant changes in the clustering structure), and (3) exploit the network heterogeneity by distributing the tasks according to the capabilities of the nodes.

The objectives of our demonstration are the following:

- Topology construction and maintenance. We show how the WSN dynamically self-organizes into a clustering structure and how this structure is maintained when the topology changes.

- Service discovery and usage. We show how the users or other devices are able to discover and use

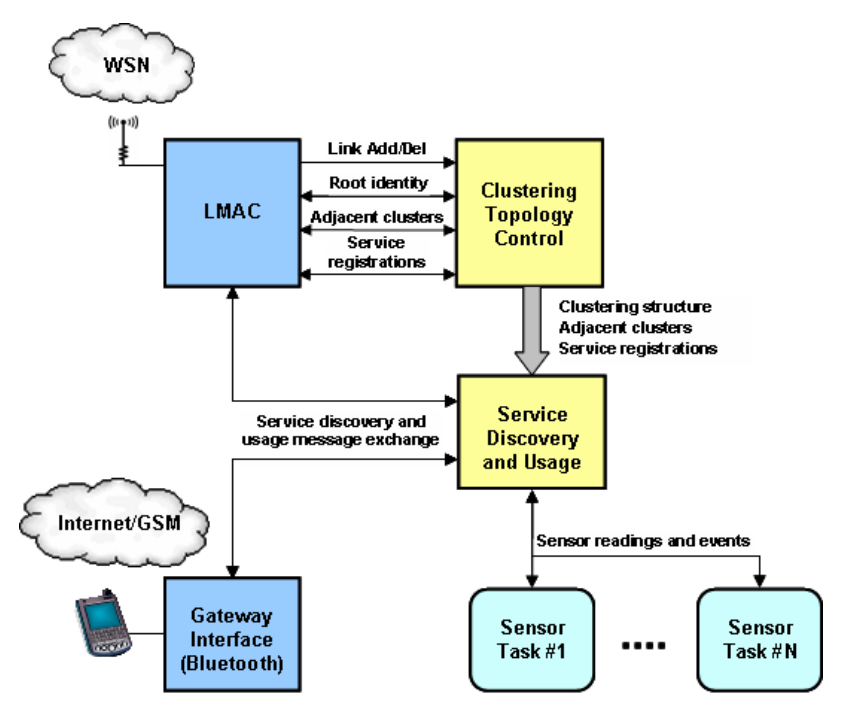

Fig. 1. Implementation overview.

the services available in the WSN, by exploiting the underlying clustering structure.

- Integration with mobile platforms. We show how the WSN can discover and use the available gateways to the outside world (mobile phones, PDAs, laptops) for signalling relevant events.

\section{IMPLEMENTATION OVERVIEW}

In the following, we give an overview of the functional modules of our prototype implementation (see Figure 1). One of the most important components is the MAC protocol, which regulates the access to the wireless medium. We use LMAC [3], a TDMA-based protocol designed for WSNs. LMAC divides time into time slots, every node being assigned one time slot to transmit the data. During its time slot, a node transmits a control packet (as a heartbeat), followed by the actual data.

The Clustering Topology Control module constructs and maintains the clustering structure. This module analyses the neighbourhood information provided by 


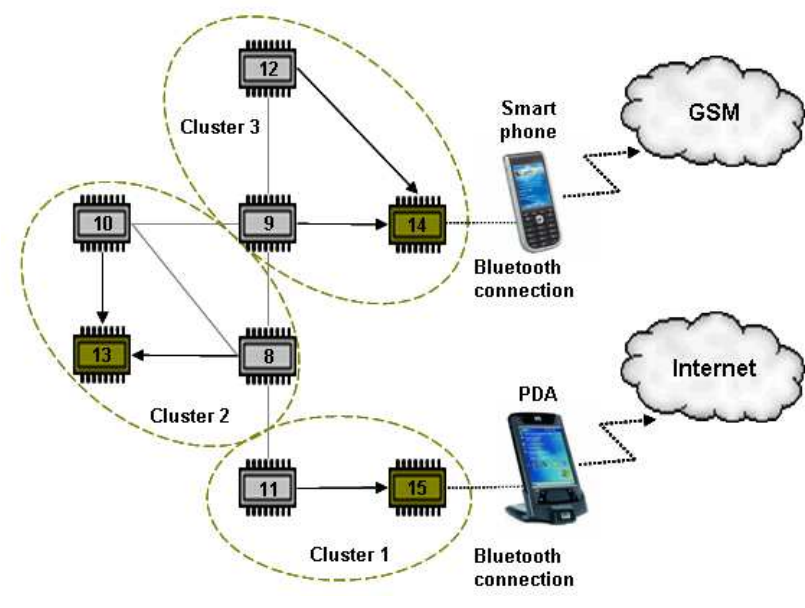

Fig. 2. Demonstration setting.

LMAC and constructs the clustering structure, by choosing the appropriate parent node. Using also the information received from the neighbours, this module maintains the root identity, knowledge on adjacent clusters and the service registrations. This information is used by the Service Discovery and Usage module in order to decide the next hop on the path to the service provider or consumer.

\section{DEMONSTRATION}

\section{A. Hardware}

We implement the service discovery protocol on the Ambient $\mu$ Node 2.0 platform [1], provided with the lowpower MSP430 micro-controller produced by Texas Instruments. The sensor node offers $48 \mathrm{kB}$ of Flash memory and $10 \mathrm{kB}$ of RAM. In our demonstration setting, nodes are equipped with light, temperature and movement (tilt switch) sensors. Two nodes incorporate Bluetooth modules in order to act as gateways to mobile devices (see Figure 2).

\section{B. Demo setting}

We demonstrate the discovery and usage of the following services: sensor readings (temperature, light, vibrations), SMS and Email.

Figure 2 shows an example setting of nine nodes which self-organize into three clusters. The dark coloured nodes $(13,14,15)$ are elected as roots since they have the highest capabilities in their neighbourhood. Two nodes act as gateways, being connected via Bluetooth to a smartphone and a PDA, which in turn connect to the GSM network and Internet, respectively.

In our demonstration, the sensor nodes which play the clusterhead role display a different LED colour than

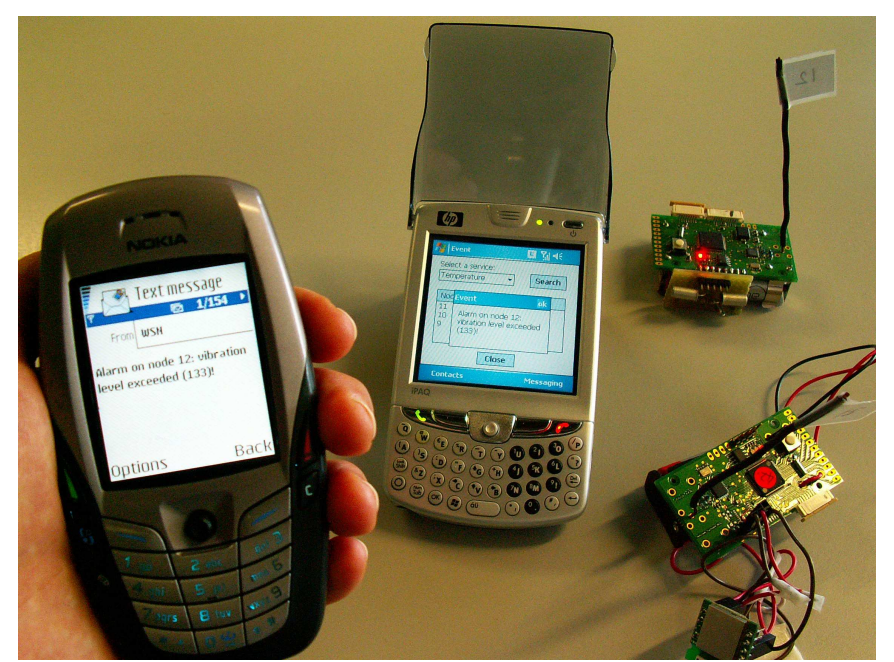

Fig. 3. Announcing events.

the ordinary nodes. By following the LED convention, we show how topological changes (adding or removing nodes from the network) trigger changes of the clustering structure. Apart from the LED convention, topological changes are transparent for the user and do not affect the functionality of the protocol.

Figure 3 shows a detail of the demonstration setting, namely two sensor nodes, a mobile phone and a smartphone. The first sensor node is equipped with a tilt switch sensor and is thus capable of detecting vibrations. The second node is provided with a Bluetooth interface and can connect to the smartphone to send events. The situation depicted in the figure is the following: the node with a tilt switch sensor detects an abnormal level of vibrations and issues a message for discovering an SMS service. The message travels the clustered WSN and reaches the node with the Bluetooth module, which provides the desired service and transmits the message to the smartphone. The smartphone in turn, displays an alarm message and sends an SMS to the mobile phone.

\section{REFERENCES}

[1] Ambient systems. http://www.ambient-systems.net.

[2] Collaborative Business Items (CoBIs). http://www.cobisonline.de.

[3] L. Van Hoesel, T. Nieberg, J. Wu, and P.J.M. Havinga. Prolonging the lifetime of wireless sensor networks by cross-layer interaction. IEEE Wireless Communications, 11(6):78-86, 2004.

[4] M. Marin-Perianu, T.J. Hofmeijer, and P. J. M.Havinga. Implementing business rules on sensor nodes. In 11th IEEE International Conference on Emerging Technologies and Factory Automation (ETFA), pages 292-299, 2006.

[5] R. S. Marin-Perianu, J. Scholten, P. J. M. Havinga, and P. H. Hartel. Energy-efficient cluster-based service discovery in wireless sensor networks. In Proceedings of the 31st IEEE Conference on Local Computer Networks, pages 931-938, November 2006. 